\title{
LAS PROPUESTAS LUDOTÉCNICAS: UNA HERRAMIENTA METODOLÓGICA ÚTLL PARA LA INICIACIÓN DEPORTIVA AL ATLETISMO EN PRIMARIA
}

Autor

\section{Resumen}

Dr. Alfonso Valero Valenzuela Facultad de Humanidades y Ciencias de la Educación Universidad de Almería

El análisis de las características de la conducta del niño a nivel cognitivo, afectivo y motor en el tercer ciclo de Primaria, se sitúa al juego dirigido como la herramienta ideal para desarrollar los contenidos deportivos en esta etapa. Los deportes individuales que están marcados por una elevada exigencia física y técnica, encuentran en las denominadas «propuestas ludotécnicas» para el aprendizaje de las disciplinas atléticas, una variante de las formas lúdicas, que destacan por un elevado nivel de compromiso de los niños con las tareas, la inclusión de reglas técnicas en las formas jugadas, un suficiente número de oportunidades para que todos los participantes puedan ponerlas en práctica, y siempre bajo un ambiente de diversión y disfrute entre los practicantes, que contribuyen de un modo integral a la formación del niño en el colegio.

Palabras clave: Educación Primaria, iniciación deportiva, juego, atletismo.

\section{Introducción}

Dentro del campo de la iniciación deportiva en el colegio, centrada especialmente en el tercer ciclo de Primaria (de 10 a 12 años), el aprendizaje de la técnica se ha venido realizando desde propuestas analíticas y repetitivas, basadas en una metodología tradicional centrada en la mejora del rendimiento, con una concepción sesgada de la conducta humana que únicamente consideraba el dominio motor y la mejora de las cualidades físicas condicionales y coordinativas, lo que ha generado insatisfacción por parte del alumnado y su exclusión del currículo de Primaria, por falta de adecuación con los actuales planteamientos pedagógicos (Valero, 2004a). Ante esta problemática, la pedagogía de iniciación a los deportes colectivos ha reaccionado dando un giro en los planteamientos metodológicos, anteponiendo el aprendizaje de la táctica y la estrategia al de la técnica, y el desarrollo de los aspectos cognitivos y socioafectivos, creando nuevos enfoques de iniciación deportiva como la enseñanza para la comprensión (Bunker y Thorpe, 1983), juegos tácticos (Mitchell, Oslin y Griffin, 2003), el enfoque estructu- ral (Blázquez, 1995) y el deporte educativo (Siedentop, 2002), entre otros.

En cambio en los deportes individuales, donde el componente táctico y estratégico es reducido y la técnica junto con las cualidades físicas condicionales son los principales factores que delimitan el desarrollo de la práctica deportiva, no se han llevado a cabo propuestas definidas que sean una alternativa eficaz al modelo tradicional de iniciación deportiva. Desde este trabajo se presenta una nueva herramienta metodológica que trata de mejorar la técnica deportiva, sin olvidar otros aspectos importantes en la conducta del ser humano como es el cognitivo y el socioafectivo.

\section{Caracteristicas de la conducta del niño en el tercer ciclo de Primaria}

Según Valero (2002), desde el actual sistema educativo, el juego puede ser entendido desde una triple perspectiva, como medio globalizador, como objeto de estudio, y como estrategia meto- 
dológica. Precisamente, esta última visión está directamente relacionada con el concepto de juego educativo y su utilización en el aprendizaje de los distintos contenidos en la Educación Física, al desarrollar los ámbitos de la conducta humana, que se dividen en el dominio cognitivo, socioafectivo y motriz (Rink, 2001).

Entre las principales características a nivel cognoscitivo, destaca que el niño entra de lleno en el subperiodo de las «operaciones concretas», donde empieza a utilizar la lógica y a ser más independiente de los aspectos puramente perceptivos, por lo que su pensamiento se vuelve mucho más móvily obedece a las leyes propias (Bueno Moral y cols. 1990). Genera procesos cognitivos más complejos y se incrementa la posibilidad de prestar atención y de relacionar simultáneamente más unidades de información. Según Papalia y Wendkos (1993), los niños llegan a ser más hábiles en el uso de estrategias de memoria tales como recitación, organización, elaboración y ayudas externas y en este sentido Martí (1999), indica que se ajusta de un modo más flexible, más eficiente y de forma más económica a las exigencias de cada situación con respecto a edades anteriores.

A nivel socioafectivo, desde el punto de vista de las emociones, los sentimientos y las actitudes, según Bueno Moral y cols. (1990), es un período de gran estabilidad y armonía, pues no existen conflictos internos de importancia en el proceso evolutivo. Para Ruiz Pérez y cols. (1990), el estado de ánimo que permanece es el de «optimismo», buen humor y alegría desbordante.

Fernández Fernández y cols. (1993); Chinchilla y Alonso Ballesteros (1998), coinciden a la hora de manifestar que durante este período aparece con fuerza el sentido de la competitividad, se evoluciona del trabajo en solitario al realizado en colaboración, el niño se vuelve extrovertido debido a su ajuste a las normas y a la buena relación con el adulto, se desarrolla el afán de aventuras y gusto por el disfrute del prestigio social, comienzan a surgir las primeras diferencias entre el comportamiento social de niños y niñas, y aparecen nuevos sentidos morales, que conducen a transformaciones en la concepción de la regla.

Siguiendo a otros autores como Gratiot-Alphandéry y Zazzo (1980), aluden que el juego social se da a partir de los 9 años teniendo dos fines: primero, asegurar el placer de los intercambios, de la exaltación de la alegría al ser compartida, y segundo, permitir a cada uno afirmarse ante los demás, y que los demás le tengan en cuenta. Moreno (1999), indica que a esta edad los niños comprenden ya el sentido de las reglas al considerarlas como prescripciones que pueden ser modificadas por consenso si todos se ponen de acuerdo, encontrando según Ruiz Pérez y cols. (1990), que las habilidades motrices desarrolladas en el juego y en partes más sistemáticas de la Educación Física mejoran los sentimientos generales de la eficiencia, favoreciendo el autoconcepto positivo.

Para Papalia y Wendkos (1993); Palacios e Hidalgo (1999), la vertiente social del autoconcepto se manifiesta de un modo más patente, haciéndose predominantes las relaciones interpersonales y las comparaciones con otros niños y niñas. En cuanto a la autoestima, Palacios e Hidalgo (1999), afirman que crece el uso de las comparaciones sociales que se van a traducir en una reducción del optimismo anterior y en una valoración cada vez más objetiva y realista.

Por último a nivel motor, hacia los diez años, según establecen Ruiz Pérez y cols. (1990), una de las mejoras que se produce en el niño es el descubrimiento del eje antero-posterior que enriquece su esquema corporal, hace que sus movimientos sean más complejos, posibilita el control de más de un gesto a la vez y permite iniciar el trabajo con movimientos analíticos. Esta idea es compartida por Oña (1987), quien añade que él gesto se consigue dividir en sus fases y en los segmentos intervinientes.

Según Ruiz Pérez y cols. (1990), a este período de edad lo denominan los expertos como uedad de oro» de los aprendizajes motores. La estabilidad en los cambios de tipo biológico crea un momento propicio para la introducción de técnicas de movimiento, tales como las que caracterizan los deportes escolares. Para Gesell y cols. (1997), constituye una edad deportiva por el puro placer que experimenta en la simple actividad física de correr, trepar, saltar, tirarse por un tobogán, patinar, andar en bicicleta. Según Bueno Moral y cols. (1990), se trata de una fase de vitalidad exuberante que busca espontáneamente el rendimiento y la competición.

Ruiz Pérez (1987), indica que las capacidades físicas evolucionan como consecuencia del desarrollo biológico y del entrenamiento. La maduración del sistema nervioso del niño permite un mayor tratamiento de las informaciones, determinando el acceso a aprendizajes cada vez más complejos, como se observa en el ámbito deportivo.

Oña (1987); Rigal (1987), informan que el progreso de la coordinación es continuo, de modo que entre los 9 y 10 años, el niño ya no sólo es capaz de orientarse a sí mismo en el espacio, sino que orienta a los objetos y a los demás respecto a sí mismo y entre ellos. Del mismo modo, es capaz de controlar las combinaciones de ritmos, lo que supone la posibilidad de captar las diferencias en determinadas tareas en cuanto a cambios de ritmo y de realizar tareas donde se combinan distintos ritmos.

A la evolución de la coordinación hay que sumarle el progreso de las habilidades como la ca- 
rrera, los saltos, combinaciones entre ambos, los lanzamientos y la recepción de objetos (Rigal, 1987: Oña, 1987; Hernández Àlvarez, 1993; Hernández Álvarez y López Crespo, 1997). Para Dick (1993), el desarrollo del juego constituye un vehículo esencial en el desarrollo total y una progresión desde un juego individual informal hasta la formalidad del deporte organizado.

Los deportes son bien aceptados siempre que guarden proporción con las facultades físicas de cada individuo y algunos deportes específicos pueden ocupar todas las horas que un niño de 9-10 años tiene ocasión de pasar al aire libre. Aún así, la iniciación deportiva ha de ser polifacética y progresiva. Se tiene que ir adaptando a la competición como fenómeno complejo y de gran carga emocional, en donde la carrera deportiva del sujeto ha de ser escalonada y piramidal, no siendo aconse jable la especialización (Oña, 1987) y sí el desarrollo de la técnica mediante el juego (Dick, 1993).

\section{El juego dirigido para la iniciación deportiva en primaria}

El juego constituye el vehículo esencial para el desarrollo integral de la persona, siendo para la mayoría de los niños la meta última en la vida (Gesell y cols. 1997), y el actual sistema educativo en Primaria está basado en el aprendizaje a través del juego (Valero, 2002). El educador ha de saber utilizar el juego para orientar e introducir las actividades deportivas dentro de un contexto educativo, siendo los principales objetivos para Blázquez (1995), el ampliar el acervo motor de las habilidades motrices y habilidades deportivas, perfeccionar y progresar en la ejecución de las mismas, fomentar una buena disposición para el posible rendimiento deportivo, favorecer la socialización-cooperación, desarrollar la emancipación y autonomía, orientar y ayudar a la adaptación deportiva, permitir la opcionalidad y encauzar hacia una posible especialización.

El juego no es exclusivamente el medio principal de desarrollo de las habilidades de los alumnos (Howarth,1989), sino que jugar es en sí mismo el principal objetivo de la iniciación (Antón y Dolado, 1997). Los enfoques alternativos, están basados sobre una forma estándar de juego deportivo que es modificada poco a poco para ayudar al estudiante a encauzar importantes conceptos y estrategias de todos los deportes (Doolittle, 1995).

De este modo, el juego se convierte en el elemento clave de los nuevos enfoques metodológicos como el comprensivo, haciéndole ver al niño el ejercicio como una actividad lúdica deportiva, más que como una práctica de habilidad determinada por el profesor que prohíbe u obstruye la práctica deportiva (Werner y cols., 1996).

Sin embargo, al referirse al término juego hay que saber distinguir entre las diferentes acepciones que posee, siendo numerosos los autores que cuando hablan del juego coinciden en distinguir entre el juego libre o espontáneo, que se realiza fuera de la influencia del profesor y el juego dirigido o didáctico, que es empleado por el profesor para aplicarlo al campo educativo y enfocado a reforzar una habilidad concreta, manipulando las reglas del juego original y reforzando unos contenidos determinados para centrarse en errores y carencias de los alumnos (Coakley, 1983; Bayer, 1986: Antón y Dolado, 1997). Para Brito (2000), se trata de una actividad propuesta, organizada y vigilada por el profesor, conteniendo una intención formativa y siendo utilizado para lograr en los niños muchos aprendizajes. Por tanto, es precisamente este juego denominado dirigido, el que mejor puede llegar a cumplir con los objetivos que han de cubrir las actividades deportivas dentro del contexto, tal y como se apuntaba anteriormente.

En esta misma línea, Méndez (1999), aporta una clasificación de los juegos, distinguiendo entre el juego libre, el juego dirigido y el juego institucionalizado o deporte, que resulta especialmente interesante por la clasificación que a su vez hace del juego dirigido, donde habla de las formas jugadas, los juegos reducidos, los juegos modificados y los minideportes, todos ellos elementos conductores del aprendizaje de los deportes (cuadro 1).

En concreto indica que los juegos reducidos son recursos didácticos fundamentados en la disminución del número de jugadores y del terreno de juego en pro de la participación y del aprendizaje de los principios deportivos. Los juegos modificados o simplificados están relacionados con los juegos reducidos, si bien, acentúan el énfasis en los aspectos tácticos y los propios alumnos pueden proponer variaciones según el rumbo del proceso de aprendizaje, siendo concebidos como recursos metodológicos. Los minideportes son adaptaciones en cuanto a la forma de los deportes institucionales para hacerlos más accesibles a las características psico-físicas de los niños, especialmente en cuanto a la reducción en el número de jugadores, terreno de juego, tamaño del móvil y de las metas, y en la variación de las reglas complejas del juego adulto, aunque se reproducen en gran medida las formas de aprendizaje centradas en la técnica.

Una variante más que distingue Méndez (1999), son las formas jugadas, que en este trabajo, muestran una especial relevancia, ya que son entendidas como una adaptación y acondicionamiento de la tarea, para que su presentación sea más acorde con una determinada etapa de aprendizaje. Se fun- 


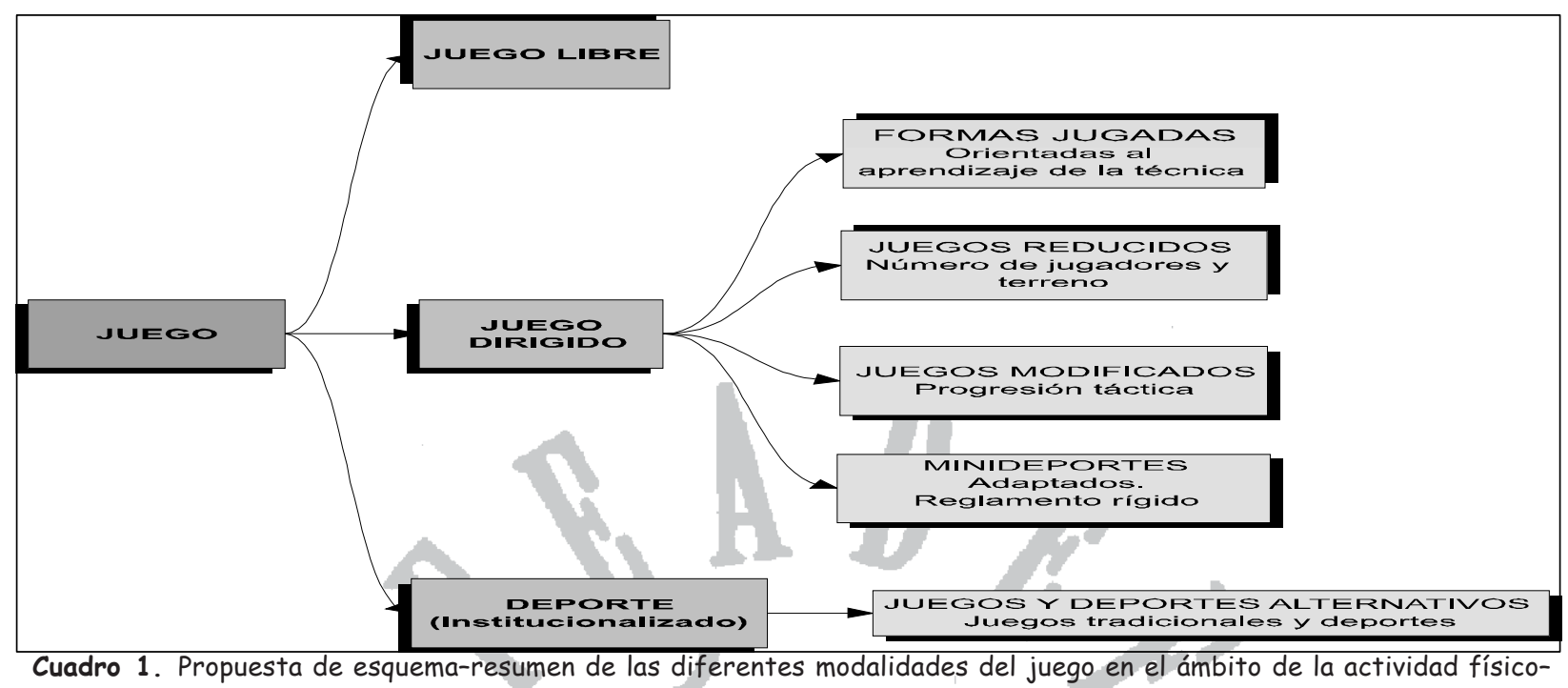
deportiva. Modificada en parte de Méndez (1999; pg. 32).

damentan en la transformación de los ejercicios confiriéndoles cierto carácter lúdico a través de un componente de reto o de una estructura competitiva y están principalmente orientadas al aprendizaje técnico y al desarrollo de las cualidades físicas y motrices, siendo interesante su utilización en la iniciación a deportes con un elevado componente técnico como es el caso de los deportes individuales.

\section{Las propuestas ludotécnicas una herrameinta en la adquisión de la técnica atlética}

Los deportes en los que la exigencia técnica es elevada, como es el caso del atletismo, es aconsejable que el contenido de aprendizaje (las disciplinas atléticas), sea dividido en pequeñas habilidades o fases para favorecer una adquisición progresiva de los patrones motores durante la realización de las tareas. Para ello, los profesores necesitan conocer las fases de las que está compuesta la disciplina atlética y así poder dividirla en una secuencia de pasos asequibles a los estudiantes a través de las formas jugadas. En este sentido, Valero y Conde (2003), elaboran un nuevo enfoque metodológico para la iniciación al atletismo denominado «Ludotécnico», que trata de ser una enseñanza alternativa a la tradicional, destacando como eje central de este enfoque las «propuestas ludotécnicas», es decir, juegos dirigidos procedentes de juegos populares, tradicionales, propios del atletismo o de otros deportes, que han sido modificados en parte al introducirle una regla técnica que hace referencia a alguno de los elementos o de las fases en las que ha sido dividida la disciplina atlética.

Las propuestas ludotécnicas pueden considerarse una variante de las formas jugadas, en cuanto a que están orientadas a la enseñanza de la técnica deportiva. En cambio, a diferencia de lo que indica Navarro Adelantado (1993) y Díaz y cols. (1995), no tienen siempre un rápido desenlace, una estructura simple, una corta duración o proceden de ejercicios a los que se les ha introducido algún elemento lúdico o competitivo.

Las principales características que reúnen las propuestas ludotécnicas son un elevado nivel de participación o grado de compromiso de los alumnos en las tareas que se están desarrollando, inclusión de alguna parte del movimiento de la disciplina que se pretende enseñar a través de una regla técnica en la forma jugada (cuña técnica), suficiente número de oportunidades para que la regla técnica sea practicada por todos los participantes, y diversión, las tareas que se realizan han de llevar a los niños a disfrutar con lo que están haciendo.

\subsection{Elevado nivel de compromiso con las tareas}

La duración de cada una de las propuestas ludotécnicas es variable, ya que a pesar de estar planificada por el profesor, sólo tiene un carácter orientativo. El tiempo que se le ha de dedicar a cada una de las propuestas está supeditado al nivel de participación de los niños con las formas jugadas, de tal modo que, si el profesor considera que los niños comienzan a perder el interés es conveniente que se pase a otra. Proporcionalmente adecuadas a su desarrollo, las propuestas han de estar adaptadas a la edad de los practicantes y a la experiencia acumulada, favoreciendo la mejora de las habilidades de los niños, no siendo una copia de las competiciones vistas en televisión, y sí permitiendo desarrollar su potencial físico (O'Neill, 1998). Tanto las instalaciones como el material 
empleado han de estar adaptados a las capacidades de los niños, mientras que las exigencias del maestro han de estar en relación con dichas capacidades. Esto se hace especialmente relevante en el grupo de disciplinas de los lanzamientos, donde el niño ha de tener sensación de que los artefactos son pesados, lo que le facilita la concienciación hacia un tipo de técnica, pero no tiene por qué ser los establecidos por las federaciones o estamentos oficiales. Los niños han de diferenciar y respetar los momentos de lanzamiento, de los de recogida para evitar accidentes. En las pruebas de carreras con obstáculos, la altura de las vallas no ha de ser un impedimento para que ellos puedan realizar las pruebas y tener la sensación del paso de un obstáculo. Y en las pruebas de saltos, se ha de tener un especial cuidado con las zonas de caídas para evitar lesiones, adaptando las exigencias en pruebas como los saltos con pértiga, triple salto y salto de altura, para fomentar más la adquisición de ritmo y coordinación, que una técnica depurada. Dentro de este bloque de disciplinas, algunas requieren un material algo más específico, como colchonetas quitamiedos en el caso de los saltos verticales, pero salvando estos inconvenientes, los alumnos pueden experimentar, si no el gesto completo, sí gran parte del mismo, evitando largas esperas, o lo que es lo mismo, logrando con las propuestas ludotécnicas un elevado tiempo de compromiso motor y de un modo seguro.

\subsection{Inclusión de reglas técnicas o también denominadas «cuñas técnicas»}

Para Conde y Valero (1997), la cuña técnica es un elemento específico que se introduce en las propuestas ludotécnicas, con objeto de que el niño jugando, incorpore y asimile las diferentes partes o fases del gesto técnico, logrando que centre la atención en el elemento que quiere trabajar y no sólo en el juego. Para ello, el profesor ha de dividir la disciplina atlética en partes, convirtiéndose cada una de estas partes en uno de los elementos específicos que contiene las propuestas ludotécnicas a través de reglas técnicas. Un ejemplo que puede ayudar a clarificar este concepto, es en el aprendizaje de las carreras de vallas, con alumnos de quinto de Primaria, el profesor divide el gesto global en tres fases que pueden ser a) aproximación, b) acción de la pierna de ataque y c) acción de la pierna de paso. A continuación, cuando el profesor explica una propuesta ludotécnica a sus alumnos, la forma jugada contiene en la mayoría de los casos una regla técnica referida a una de estas tres fases en las que se ha dividido el gesto técnico de la disciplina «carreras de vallas». En la propuesta ludotécnica denominada «el paso de la cuerda» (Valero y Conde, 2003), donde la clase se divide en grupos de 6-8 alumnos formando un círculo, separados un metro unos de otros y orientados mirando hacia la espalda del compañero, un jugador se coloca en el centro con una cuerda en cuyo extremo hay una pelota metida en una bolsa de plástico, y da vueltas en sentido frontal a la orientación de los jugadores, mientras que el resto de los compañeros saltan la pelota. La regla que todos han de cumplir es cuando saltan que extiendan al frente la pierna que primero se despega del suelo, inclinando el tronco hacia delante. Esta regla técnica está en conexión con una de las tres fases en las que ha dividido la disciplina atlética, en concreto con la b) acción de la pierna de ataque, siendo precisamente este requerimiento (también denominado «cuña técnica»), el que logra que el niño a través del juego adquiera la técnica deportiva (cuadro 2).

La dificultad de las propuestas ludotécnicas varía en función del nivel de aprendizaje de los niños, siendo mayor el número de fases o componentes en los que se divide una disciplina conforme aumenta la edad. Además, la complejidad de una misma propuesta ludotécnica depende del número de cuñas técnicas que incluya. En las primeras sesiones interesa que las formas jugadas estén compuestas por una única regla técnica, pero en sesiones más avanzadas se pueden incluir dos. La intención es facilitar el aprendizaje del niño tanto a nivel motor, creando un nuevo patrón de movimiento, como a nivel cognitivo, siendo capaz de centrar la atención en el elemento técnico introducido, para que en sesiones posteriores sea capaz de identificar cuáles son las reglas técnicas y centrar la atención en más de un elemento técnico, mientras lleva a cabo el juego. El dominio cognitivo y motor están vinculados, debiéndose plantear si cuando el niño no ejecuta bien la habilidad se debe a que no la entiende o a que no es capaz de ejecutarla.

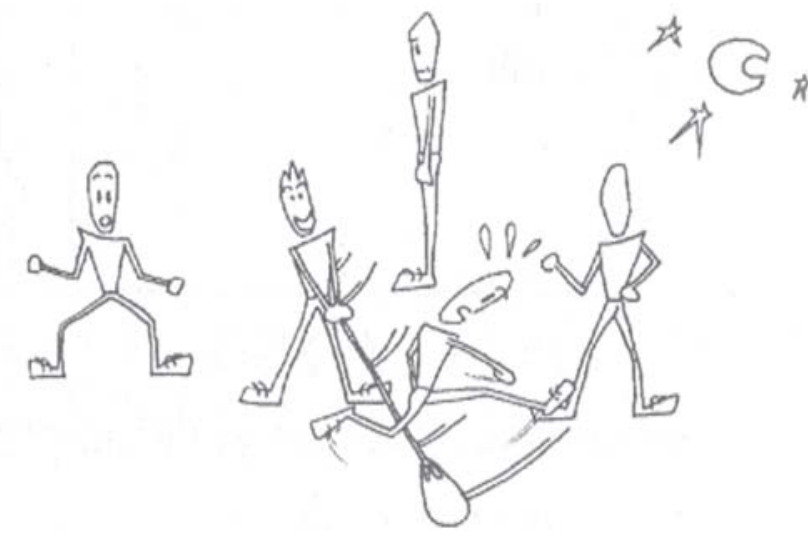

Cuadro 2. Juego «el paso de la cuerda», extraído de Valero y Conde (2003; pg. 154). 


\subsection{Suficiente número de oportunidades}

Estas formas jugadas que incluyen reglas técnicas han de proporcionar un número suficiente de intentos para que todos y cada uno de los niños lleven a cabo la cuña técnica que está en conexión directa con una de las partes del gesto atlético que se está enseñando. Por tanto, la propuesta ludotécnica ha de asegurar al menos una ejecución del elemento técnico a todos los alumnos. Estrategias para lograrlo pueden ser disminuir el número de componentes que integran los equipos, e incrementar la cantidad la material, no existiendo una agrupación característica en las propuestas ludotécnicas.

En este sentido, el docente ha de ser consciente de que siempre que se están realizando las propuestas ludotécnicas hay que considerar que se está trabajando parte de las habilidades requeridas en el gesto global de la disciplina atlética, y por tanto, se persiguen situaciones durante la práctica de los juegos que involucren a todos los niños en las actividades, ejecutando el movimiento de manera adecuada. Las diferencias físicas no se convierten en un componente discriminatorio o un obstáculo en la progresión de los elementos técnicos del movimiento, debiendo mostrar el profesor escasa preocupación por el trabajo de las cualidades físicas condicionales (Valero, 2004b).

En el ejemplo utilizado con la propuesta ludotécnica de «el paso de la cuerda», todos los componentes del grupo, excepto quien hace girar la cuerda con la pelota enganchada al extremo, realizan la «cuña técnica» indicada con cada giro completo al círculo formado por los niños, siendo una cuestión importante a tener en cuenta el profesor, que cada cierto tiempo, sea otro el niño que tenga que girar la pelota, para que de este modo, se asegure que todos hayan ejecutado el elemento técnico que se perseguía trabajar cuando fue confeccionada la propuesta ludotécnica.

\subsection{Diversión}

El uso de formas jugadas va a asegurar la diversión durante el desarrollo de las tareas, consiguiendo que sea mucho más motivante la sesión de iniciación al atletismo. Utilizando el juego como herramienta de trabajo durante el avance de las propuestas ludotécnicas, se provoca un aumento de la diversión, incidiendo de forma directa sobre la motivación de los niños y contactando con sus intereses para alcanzar un aprendizaje óptimo (Vale- ro, 2005). El juego es el elemento central del proceso de enseñanza, siempre presente y del que parte el aprendizaje, provocando que las prácticas sean alegres y divertidas. Con él, se trata de introducir una rica variedad de formas jugadas de acuerdo con la edad y la experiencia de los niños, además de cómo apunta Howarth (1989), ayudar a superar la inapropiada enseñanza de patrones de calentamiento, práctica y juego. El calentamiento entendido como un conjunto de ejercicios de desplazamiento, movilidad articular, activación de grandes masas musculares y estiramientos, es suprimido en el enfoque Ludotécnico, por las propuestas ludotécnicas, sabiendo que el objetivo de evitar lesiones se cumple con una actuación prudente del docente, que regula la intensidad de las propuestas, comenzando con las de menor intensidad y dificultad para ir progresivamente incrementando la complejidad técnica y exigencia física, pasando por todas las posibles combinaciones en las agrupaciones, desde individual, parejas, pequeños y grandes grupos, hasta toda la clase, lo cual es un elemento a favor de la comunicación entre los compañeros de clase, que fomenta las relaciones sociales (Valero, 2004b).

Según Oslin (1996), una aproximación a la iniciación deportiva centrada en la técnica constriñe la capacidad creativa de los participantes, reduciendo la posibilidad de que un amplio número de alumnos disfruten del juego, generando insatisfacción cuando ocurren bajo condiciones controladas que se apoyan poco, o no tienen relación alguna con el juego.

El material empleado suele ser alternativo, fácil de hallar en el almacén del gimnasio y utilizado en la pista polideportiva donde se desarrolla la sesión, procurando que sea lo más variado posible, buscando el máximo enriquecimiento y bagaje motriz por parte de los niños. Tampoco se descarta la utilización de un material más convencional como bolas de caucho que evitan deteriorar el suelo de la instalación deportiva o vallas antilesión, recubiertas de corcho, que aportan más seguridad al niño en la realización de la práctica. En cualquiera de los casos, el material ha de estar adaptado al niño, siendo tentador y atrayente, incitando a los niños a su descubrimiento, uso y manipulación.

Por último, las instalaciones necesarias para poder llevar a cabo las propuestas ludotécnicas son las mismas que las empleadas para cualquier actividad físico deportiva, siendo este hecho una de las grandes ventajas que poseen las propuestas ludotécnicas frente a los ejercicios tradicionalmente empleados en la iniciación al atletismo. Por tanto, pueden ser puestas en práctica en gimnasios, pistas polideportivas y por supuesto, en una pista de atletismo, sin que el medio donde se desarrollen sea un impedimento para su puesta en escena. 
A modo de conclusión resaltar que desde el contexto educativo de la educación Primaria, el aprendizaje de la técnica deportiva no ha de ser entendido como un compartimento estanco, sino en conexión con los dominios cognitivo y afectivo, contribuyendo todos ellos a la formación integral de la persona. Las propuestas lúdicas se han presentado como una herramienta útil para lograrlo, tanto por su elevado tiempo de implicación en la tarea, favoreciendo la inclusión de todo el alumnado en las actividades, como por la búsqueda de un incremento en el grado de diversión sin olvidar los elementos técnicos de las disciplinas atléticas, que logran a través de las cuñas técnicas que el niño jugando, asimile los patrones del movimiento deportivo. El dominio de la habilidad deja de ser el único objetivo de la práctica deportiva, para adquirir un papel más relevante el dominio afectivo y cognitivo, que de manera conjunta contribuyen a la formación completa del niño dentro del colegio

\section{Referencias bibligráficas}

Antón, J. y Dolado, M. M. (1997). La iniciación a los deportes colectivos: una propuesta metodológica. En J. Giménez, J.P. Sáenz-López, y M. Díaz Trillo. El deporte escolar. Universidad de Huelva.

Bayer, C. (1986). La enseñanza de los juegos deportivos colectivos: baloncesto, fútbol, balonmano, hockey sobre hierba y sobre hielo, rugby, balonvolea, waterpolo. Barcelona: Hispano Europea.

Blázquez, D. (1995). Métodos de enseñanza de la práctica deportiva. En D. Blázquez, La iniciación deportiva y el deporte escolar. Barcelona: Inde.

Brito, L.F. (2000). El juego: una propuesta metodológica activa. Lecturas: Educación Física y Deportes. www.efdeportes.com/Revista Digital. 5, 22.

Bueno Moral, M. L., Campuzano, J., Fontecha, C., Manchón, J.O., Moral García, P., Perona, M., Rico Pérez, I., Ruiz Pérez, L.M. y Vázquez Gómez, B. (1990). Educación Primaria. Educación Física. Segundo Ciclo: 8 a 10 años. Madrid: Gymnos.

Bunker, D. y Thorpe, R. (1983). A Model for the Teaching of Games in Secondary Schools. Bulletin of Physical Education, 19, 5-8.

Chinchilla, J. L. y Alonso Ballesteros, J. (1998). Educación Física en Primaria. Segundo Ciclo / 1. Madrid: CCS.

Coakley, J. J. (1983). Play, Games, and Sport: Developmental Implications for Young People. En
Harris y Park. (Comps.) Play, Games \& Sports in cultural contexts. Champaign: Human Kinetics Books.

Conde, J. L. y Valero, A. (1997). Modelo Renovador de Iniciación Deportiva al Atletismo. Ciencias de la Actividad Física. 5, 109-121.

Díaz Trillo, M., Sáenz-López, P. y Tierra, J. (1995). Iniciación Deportiva en Primaria: Actividades Físicas Organizadas. Sevilla: Wanceulen.

Dick, F. (1993). Principios del Entrenamiento Deportivo. Barcelona: Paidotribo.

Doolittle, S. (1995). Teaching Net Games to Low Skilled Students: A Teaching for Understanding Approach. The Journal of Physical Education, Recreation \& Dance, 66, 18-23.

Fernández Fernández, J. P., García Díaz, R. y Posada, F. (1993). Guía para el Diseño Curricular en Educación Física. Lérida: Agonos.

Gesell, A., Ilg, F. L. y Bates, L. (1997). El adolescente de 10 a 16 años. Barcelona: Paidós.

Gratiot-Alphandéry, H. y Zazzo, R. (1980). Tratado de psicología del niño. Tomo V. La formación de la personalidad. Madrid: Morata.

Hernández Álvarez, J. L. (1993). Aprendizaje y desarrollo motor. En T. Lleixá. La Educación Física en Primaria Reforma. (6 a 12 años). Guía del profesor. Vol. I. Barcelona: Paidotribo.

Hernández Álvarez, J. L. y López Crespo, C. (1997). Aprendizaje y desarrollo motor. En F. J. Castejón. Manual del Maestro Especialista en Educación Física. Madrid: Pila Teleña.

Howarth, K. (1989). Games Teaching. Bulletin of Physical Education, 25, 29-35.

Martí, E. (1999). Procesos cognitivos básicos y des arrollo intelectual entre los 6 años y la adolescencia. En J. Palacios, A. Marchesi, y C. Coll. Desarrollo psicológico y educación. Psicología evolutiva. Madrid: Alianza.

Méndez, A. (1999). Análisis comparativo de las técnicas de enseñanza en la iniciación a dos deportes de invasión: el floorball patines y el baloncesto. Tesis Doctoral Inédita.

Mitchell, S., Oslin, J. y Griffin, L. (2003). Sport foundations for Elementary Physical Education: a tactical games approach. Champaign: Human Kinetics.

Moreno, M. C. (1999). Desarrollo y conducta social de los 6 años a la adolescencia. En J. Palacios, A. Marchesi, y C. Coll. Desarrollo psicológico y educación. Psicología evolutiva. Madrid: Alianza.

Navarro Adelantado, V. (1993). El juego infantil. En V. Navarro Adelantado, M. J. De la Rica, F. 
Gil, y E. Estapé. Fundamentos de Educación Física para enseñanza primaria. Vol II. Barcelona: Inde.

O'Neill, J. (1998). Challenging Athletics at Key Stages 3 and 4 . The British Journal of Physical Education, 29, 13-17.

Oña, A. (1987). Desarrollo y motricidad: fundamentos evolutivos de la educación física. Granada: C.D. INEF.

Oslin, J. L. (1996). Tactical Approaches to Teaching Games. The Journal of Physical Education, Recreation \& Dance, 67, 1, 27.

Palacios, J. e Hidalgo, V. (1999). Desarrollo de la personalidad desde los 6 años hasta la adolescencia. En J. Palacios, A. Marchesi, y C. Coll. Desarrollo psicológico y educación. Psicología evolutiva. Madrid: Alianza.

Papalia, D. E. y Wendkos, S. (1993). Desarrollo Humano. Colombia: McGraw-Hill Interamericana.

Rigal, R. (1987). Motricidad humana. Fundamentos y aplicaciones pedagógicas. Madrid: Pila Teleña.

Rink, J. (2001). Investigating the assumptions of Pedagogy. Journal of Teaching in Physical Education, 20, 112-128.

Ruiz Pérez, L. M. (1987). Desarrollo motor y actividades físicas. Madrid: Gymnos.

Ruiz Pérez, L. M., Bueno Moral, M. L., Fernández Iriarte, M. J., Manchón, J. O., Moral García, P. y Saura, J. (1990). Educación Primaria. Educación Física. Primer Ciclo: 6 a 8 años. Madrid: Gymnos.

Siedentop, D. (2002). Sport education: a retrospective. Journal of Teaching in Physical Education, 21, 409-418.

Valero, A. (2002). El juego en la Educación Primaria. En J.A. Moreno, Aprendizaje a través del juego. Málaga: Aljibe.

Valero, A. (2004a). Situación actual de la metodología en la iniciación deportiva al atletismo. Revista de Educación Física. Renovar la teoría y la práctica, 94, 13-20.

Valero, A. (2004b). El enfoque ludotécnico como alternativa a la enseñanza tradicional del atletismo en la educación primaria. Aula de Encuentro, 8, 119-131.

Valero, A. (2005). Análisis de los cambios producidos en la metodología de la iniciación deportiva. Apunts, Educación Física y Deportes, 79, 5967.

Valero, A. y Conde, J. L. (2003). La iniciación al atletismo a través de los juegos (El enfoque ludotécnico en el aprendizaje de las disciplinas atléticas). Málaga: Aljibe.

Werner, P., Thorpe, R. y Bunker, D. (1996). Teaching Games for Understanding. Evolution of a Model. The Journal of Physical Education, Recreation \& Dance, 67, 28-33.

\section{$10^{\circ}$ aniversario FEADEF Albergue "EMBALSE DE ALARCÓN"}

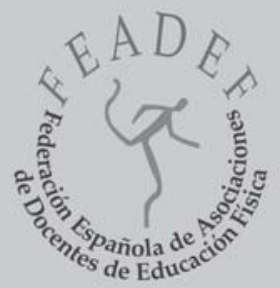

El fin de semana comprendido entre los días 8 y 10 de septiembre de 2006 tendrá lugar un ENCUENTRO conmemorativo de los 10 años de nuestra Federación.

Este encuentro tendrá lugar en un lugar admirable como es el Albergue "EMBALSE DE ALARCÓN"

Situado en el termino de Alarcón entre Valencia y Madrid, es un lugar idóneo para el relax y disfrute de los adultos y para la práctica de actividades estimulantes y recreativas para estudiantes durante todo el año.

Los que tengamos la suerte de reunirnos podremos disfrutar, además de recuerdos revitalizadores, de un paraje maravilloso que disfrutaremos muy gratamente. 